\title{
Modelação multinível e delineamento longitudinal-misto na pesquisa em Educação Física e Ciências do Esporte
}

CDD. 20.ed. 001.422

796.03

http://dx.doi.org/10.1590/1807-55092015000100035

\author{
Michele Caroline de SOUZA* \\ Cláudia Lúcia de Moraes FORJ AZ** \\ Thayse Natacha GOMES* \\ Fernanda Karina dos SANTOS* \\ J oey EISENMANN ${ }^{* * *}$ \\ J osé António Ribeiro MAIA*
}

*Faculdade de Desporto, Universidade do Porto - Portugal. **Escola de Educação Física e Esporte, Universidade de São Paulo.

***Department of $\mathrm{Ra}$ diology, Michigan State University - USA.

\section{Resumo}

0 propósito deste estudo é apresentar o uso da modelação multinivel (MMN) com informação oriunda de um estudo longitudinal-misto sobre o crescimento, desenvolvimento e saúde de adolescentes portugueses. A amostra foi composta por 5155 individuos divididos em quatro coortes que abrangem as faixas etárias dos 10 aos 12, 12 aos 14, 14 aos 16 e 16 aos 18 anos. As variáveis de estudo foram a força de preensão manual e o percentual de gordura total (\%GTotal). A análise mostrou trajetórias curvilíneas do desempenho de força de preensão, com maiores incrementos anuais nos meninos. 0 \%GTtotal apresentou associação positiva com o desempenho da força estática; constatou-se uma forte variabilidade interindividual. 0 recurso ao delineamento longitudinal-misto permitiu a recolha mais célere da informação; e a metodologia MMN mostrou-se suficientemente flexivel e robusta para acomodar, num modelo único, aspectos da mudança individual e das diferenças interindividuais do desempenho da força estática condicionados à ação do tempo.

PalavRas-Chave: Estudos longitudinais; Modelação hierárquica; Mudança; Desempenho motor.

\section{Introdução}

Essencialmente, a pesquisa em Educação Física e Ciências do Esporte centrada num delineamento longitudinal pretende responder a dois aspetos importantes de uma característica qualquer condicionada à ação do tempo - sua mudança e estabilidade. Este delineamento envolve, basicamente, a monitorização, descrição, análise e interpretação do processo de mudança de uma variável representada de modo contínuo ou discreto ao longo do tempo ${ }^{1-4}$. Para esta análise, exigem-se observaçóes repetidas e ordenadas no tempo, nem sempre equidistantes e balanceadas, de um indivíduo ou grupo de indivíduos, cujos intentos são: a) identificar diretamente a mudança intraindividual; b) marcar as diferenças ou similaridades (heterogeneidade "versus" homogeneidade) interindividuais no curso da mudança intraindividual; c) analisar as inter-relaçôes entre classes de comportamentos intraindividuais observados durante a mudança; d) analisar possíveis determinantes/"causas" da mudança intraindividual; e e) analisar as possíveis determinantes/"causas" das diferenças interindividuais na mudança intraindividual ${ }^{1}$.

O delineamento longitudinal puro (i.e., concebido para registar e analisar as mesmas variáveis, sempre nos mesmos indivíduos em intervalos de tempo específicos e equidistantes, por um período de tempo considerado aceitável ${ }^{5-8}$ ) tem um vastíssimo potencial. É habitualmente considerado o mais eficiente para o estudo de diferentes aspectos do crescimento físico humano e do desenvolvimento motor e cognitivo ${ }^{5-8}$. Não obstante a riqueza interpretativa do enorme manancial de dados que este delineamento gera, contém um conjunto de problemas que limitam, de algum modo, o seu uso corrente na pesquisa em Educação Física e Ciências do Esporte, nomeadamente: restriçóes temporais (tempo prolongado de recolha da informaçâo que 
se estende por vários anos); perda ou "drop out" amostral, muitas vezes seletivo; custos associados às mediçōes contínuas e sistemáticas; dificuldade em manter uma equipe de investigadores/avaliadores por um longo período de tempo; entre outros fatores reportados na literatura'. Uma alternativa viável a este tipo de delineamento é designando de longitudinal-misto que mantém intactas as virtualidades do delineamento longitudinal puro e minimiza as suas desvantagen $s^{5,9}$.

O delineamento longitudinal-misto, também designado de recolha acelerada da informação longitudinal, parece ter sido inicialmente proposto por $\mathrm{BELL}^{6-7}$. Caracteriza-se pela informação longitudinal de múltiplas coortes independentes de sujeitos temporariamente sobrepostas ${ }^{9}$, ou seja, este delineamento liga segmentos adjacentes de dados longitudinais de curta duração provenientes das diferentes coortes. Dentre as suas vantagens constam: a) o período relativamente curto de acompanhamento dos sujeitos, reduzindo os problemas cumulativos de efeito de re-testagem e "drop out" amostral; b) os resultados da pesquisa podem ser produzidos mais celeremente; c) a utilização de diferentes coortes aumenta a confiança na generalização dos resultados; e d) a possibilidade em estimar, sempre que necessário, a magnitude dos efeitos da idade, coorte e período de medição na análise da mudança ${ }^{6-7,9}$.

A utilidade e versatilidade do delineamento longitudinal-misto na investigação da mudança intraindividual e das diferenças interindividuais tem sido bastante fértil em diferentes áreas do desenvolvimento humano - da Psicologia à Auxologia, da Epidemiologia até à Saúde Pública ${ }^{5,8-9}$. Não obstante a sua relevância, o seu uso não parece estar muito difundido no espaço da lusofonia, principalmente no domínio da pesquisa em Educação Física e Desporto. Tal como referido por SiLva et al. ${ }^{2}$, só foi possível localizar dois

\section{Método}

\section{Amostra}

Os dados utilizados são provenientes de um estudo longitudinal-misto em curso na Regiāo do Grande Porto, Portugal, que foi genericamente desenhado para investigar a interação entre características individuais, fatores ambientais e estilos de vida no crescimento físico, desempenho motor, estilos de vida e indicadores da saúde de adolescentes estudos realizados em Portugal ${ }^{10-11}$ e outros dois no Brasil $^{12-13}$ que recorreram a este delineamento.

Nunca será demais mencionar os desafios conceituais, metodológicos e analíticos que enfrentam os pesquisadores da "coisa desenvolvimentista" muito bem descritos por Collins ${ }^{14}$. A sua posição pode ser sumariada em três etapas sequenciais: a) o recurso a um modelo teórico bem articulado para interpretar a mudança; b) servido por um delineamento temporal que permita uma visão clara e detalhada do fenômeno em estudo, a que se associa; c) um modelo estatístico suficientemente elegante, flexível, eficiente e robusto para tratar a informação disponível. Tal como bem referem Bryk e Raudenbush ${ }^{15}$, a Modelação Multinível (MMN) apresenta-se como forte candidata para responder aos desafios enunciados por Collins ${ }^{14}$, em 2006, face à sua versatilidade em permitir analisar e interpretar, de modo sólido e coerente, a dinâmica da mudança intraindividual e das diferenças interindividuais, bem como a possibilidade de incluir variáveis mediadoras e preditores fixos e/ou dinâmicos a que se associa um modo adequado de lidar com os "missings by design" gerados pelo delineamento longitudinal-misto ${ }^{3,14,16-17}$.

Não obstante a divulgaçáo no espaço da língua Portuguesa da MMN em variados domínios, bem como a existência de vários tutoriais ${ }^{3,17-18}$, a sua utilização náo é ainda corrente na pesquisa em Educação Física e Ciências do Esporte, sobretudo a que decorre do uso de delineamentos longitudinais-mistos. Daqui que o grande propósito deste texto seja o de apresentar um estudo longitudinal-misto no vasto domínio de confluências entre Educação Física/ Auxologia/Ciências do Esporte/Epidemiologia/ Saúde Pública em curso em Portugal, bem como o uso esclarecido da MMN para analisar a informação decorrente da sua aplicação. Trata-se, em suma, de uma visita guiada com um "manual de instruçôes".

portugueses com idades compreendidas entre 10 e 18 anos. Esta pesquisa, em fase de conclusão, pretende analisar adolescentes amostrados aleatoriamente de modo estratificado, divididos em quatro coortes, avaliados anualmente durante três anos consecutivos. A primeira coorte foi acompanhada dos 10 aos 12 anos; a segunda dos 12 aos 14 anos; a terceira dos 14 aos 16 anos, e a quarta dos 16 aos 18 anos. A sobreposiçáo temporal das coortes é feita aos 12, 14 e 16 anos de 
idade (TABELA 1). Para o presente estudo foram considerados os dados obtidos de um total de 5155 indivíduos (TABELA 2), e as variáveis eleitas em todas as análises foram a força de preensão manual (avaliada através de um dinamômetro portátil da marca Takei Physical Fitness Test GRIP-D) seguindo os critérios da bateria de testes da AAHPER Youth Fitness Test ${ }^{19}$, e o percentual de gordura corporal total (avaliado com um aparelho de impedância bioeléctrica, TANITA BC-418 MA, com precisão de $0,1 \%)$. O projeto e os protocolos de avaliaçáo foram aprovados pelo Conselho Científico da Faculdade de Desporto da Universidade do Porto, Portugal. As avaliaçôes foram realizadas somente após a assinatura do consentimento livre e esclarecido pelos pais e/ou encarregados de educaçáo dos adolescentes.

TABELA 1 - Estrutura básica do delineamento longitudinal-misto de acordo com as quatro coortes de idade.

\begin{tabular}{|c|c|c|c|c|c|c|c|c|c|}
\hline Coorte 1 & 10 & 11 & 12 & & & & & & \\
\hline Coorte 2 & & & 12 & 13 & 14 & & & & \\
\hline Coorte 3 & & & & & 14 & 15 & 16 & & \\
\hline Coorte 4 & & & & & & & 16 & 17 & 18 \\
\hline
\end{tabular}

TABELA 2 - Dimensão da amostra em função da idade, coorte (C1 a C4) e sexo.

\begin{tabular}{lccc}
\hline Coorte & Idades & Meninos- Meninas & Total \\
\hline C1 & $10-12$ & $532-533$ & 1065 \\
C2 & $12-14$ & $806-703$ & 1509 \\
C3 & $14-16$ & $639-705$ & 1344 \\
C4 & $16-18$ & $573-664$ & 1237 \\
\hline & Total & $2550-2605$ & 5155 \\
\hline
\end{tabular}

\section{Controle da qualidade da informação}

A eficiência e o alcance dos resultados de uma pesquisa qualquer repousam, em certa medida, nos distintos processos de controle da qualidade da informação. No presente estudo este processo foi realizado em diferentes etapas: 1) treino da equipe de "campo" por avaliadores experientes do Laboratório de Cineantropometria e Estatística Aplicada da FADE-UP, Portugal; 2) realização de retestes em subconjuntos aleatórios de sujeitos em cada dia de avaliação ao longo de todo o processo de coleta dos dados; 3 ) controle da entrada informatizada da informação e análise exploratória para identificar possíveis erros e/ ou a presença de "outliers"; e 4) cálculo de estimativas de fiabilidade baseado no coeficiente de correlação intraclasse $(\mathrm{R})$, sendo os valores obtidos: teste de força de preensão manual, $\mathrm{R}=0,97$; percentual de gordura corporal total, $\mathrm{R}=0,99$.

\section{Procedimentos estatísticos}

Tal como referido anteriormente, a análise exploratória (gráfica e numérica) da informaçáo foi realizada no "software" SPSS 20.0. A modelação das mudanças na força de preensão foi efetuada no "software" HLM 7.01", através da técnica da $\mathrm{MMN}^{15,21}$.

AMMN é, simultaneamente, um modo de pensar ${ }^{22}$ e uma metodologia elegante, robusta e extremamente flexível na análise de informação com uma estrutura designada de multinível ${ }^{23}$. Quando aplicada a dados longitudinais, permite integrar de modo coerente, num modelo estatístico relativamente complexo, a análise da mudança e variação intraindividual, à estimação da trajetória média do grupo (de sujeitos), a que se associa a inclusão da análise de variáveis preditoras ou correlatas (fixas e/ou dinâmicas) das diferenças interindividuais na mudança $\mathrm{da}(\mathrm{s})$ variável(eis) em estudo ${ }^{15}$. De fato, não há necessidade de haver uma única variável dependente, bem pelo contrário, é possível incluir várias em simultâneo. Essencialmente, a estrutura analítica da MMN encontra-se distribuída por níveis ou estratos organizacionais da informação. Qualquer número de níveis pode ser apresentado, embora a utilizaçáo mais frequente em delineamentos longitudinais seja de dois níveis - medidas repetidas e sujeitos (do inglês "repeated observations nested within subjects") ${ }^{15,24-25}$. No primeiro nível informacional de um delineamento 
longitudinal-misto formula-se um modelo para descrever a mudança, linear ou não, definido por um conjunto de parâmetros que descrevem as trajetórias individuais (submodelo intraindividual ou de medidas repetidas). No segundo nível assumese que as estimativas desses parâmetros individuais variam em função de um conjunto de preditores dos sujeitos (submodelo interindividual) ${ }^{15,24-25}$. Essa interdependência entre os parâmetros do primeiro e do segundo nível é, precisamente, o coração da estrutura hierárquica, a que se associa, essencialmente, a estimação com métodos estatísticos robustos de uma parte fixa e aleatória da $\mathrm{MMN}^{24,26}$.

No submodelo intraindividual, ou de medidas repetidas ( $1^{\circ}$ nível), a trajetória da mudança pode ser escrita, por exemplo, por uma função polinomial do tempo:

$$
\mathrm{Y}_{\mathrm{ti}}=\beta_{0 \mathrm{i}}+\beta_{1 \mathrm{i}} \mathrm{T}_{\mathrm{ti}}+\beta_{2 \mathrm{i}} \mathrm{T}_{\mathrm{ti}}^{2}+\ldots+\beta_{\mathrm{pi}} \mathrm{T}_{\mathrm{ti}}^{\mathrm{g}}+\mathrm{e}_{\mathrm{ti}}
$$

Em que $Y_{\text {ti }}$ representa a variável dependente para o indivíduo "i" na ocasião " $t$ "; os $\beta$ s são coeficientes da função polinomial de grau $\mathrm{p}\left(\beta_{0 \mathrm{i}}\right.$ é o valor no "intercept" ou "baseline" - cuja métrica temporal é habitualmente centrada no início do estudo, e daqui a atribuição de significado ao valor 0 ; os $\beta_{11}, \beta_{2 i}, \ldots, \beta_{\mathrm{pi}}$ são os parâmetros descritores da trajetória de mudança indivíduo “i”); o e é o erro aleatório. A variável preditora do nível 1 é o tempo (do inglês "time as a predictor"), representado na equação por $T$,' podendo, por exemplo, ser descrito pela idade do indivíduo "i” no tempo "t". A potência $\mathrm{T}_{\mathrm{ri}}^{\mathrm{g}}($ onde $\mathrm{g}=1,2, \ldots, \mathrm{p})$ representa a ordem da função polinomial, especificamente linear, quadrática, cúbica, ou de maior complexidade. Por exemplo, na trajetória de mudança intraindividual do sujeito $\mathrm{i}$, descrita por um polinômio de segundo grau, teríamos três parâmetros: o valor no "baseline" $\left(\beta_{0 i}\right)$, a velocidade $\left(\beta_{1 i}\right)$ e a aceleração $\left(\beta_{2 \mathrm{i}}\right)^{15,17,24-26}$.

Tal como anteriormente referido na equaçáo (1) os valores dos parâmetros descritores das trajetórias de mudança (i.e. os $\beta$ s) variam entre os indivíduos ${ }^{15-17,24-26}$. Ora no submodelo do $2^{\circ}$ nível, há que modelar a heterogeneidade das trajetórias, sendo que os parâmetros do primeiro nível passam a ser as variáveis dependentes (do inglês "intercepts and slopes as outcomes"):

$$
\begin{gathered}
\beta_{0 \mathrm{i}}=\gamma_{00}+u_{0 \mathrm{i}} \\
\beta_{1 \mathrm{i}}=\gamma_{10}+u_{1 \mathrm{i}} \\
\cdots \\
\beta_{\mathrm{pi}}=\gamma_{\mathrm{p} 0}+\mathrm{u}_{\mathrm{pi}} .
\end{gathered}
$$

$\mathrm{Na}$ equação (2), o valor do "baseline" $\left(\beta_{0 i}\right)$ e os coeficientes de regressão associados à trajetória do sujeito "i" $\left(\beta_{1 i}, \ldots, \beta_{\mathrm{pi}}\right)$ podem ser decompostos em duas partes: uma que representa a trajetória média da mudança de todos os indivíduos (a parte de efeitos fixos do modelo - do inglês "fixed effect": $\gamma_{00}=$ média no "baseline", e $\gamma_{10}, \ldots, \gamma_{\mathrm{p} 0}$ representam as componentes da trajetória média que pode ser o declive ou curva em funçáo da complexidade do polimônio); a outra parte, designada de aleatória (do inglês "random effects": $u_{0 i}, u_{1 i}, \ldots, u_{\mathrm{pi}}$ ) expressam variabilidade no baseline, bem como heterogeneidade no trajeto de mudança, i.e., os desvios dos parâmetros do indivíduo "i" relativamente à trajetória média.

No exemplo que apresentaremos de seguida, descreveremos as etapas mais importantes do uso da MMN na análise e interpretação dos dados do nosso delineamento.

\section{Variáveis e problema em estudo}

No nosso exemplo, um total de 5155 adolescentes, divididos em quatro coortes, foram avaliados anualmente, durante três anos consecutivos (20112013) no teste de força estática de preensão manual e percentual de gordura corporal total (\%GTotal). Trata-se de um delineamento relativamente complexo na sua organizaçáo e análise, pois além de descrever o comportamento do desempenho na prova de preensão manual em função do tempo (i.e. mudança intraindividual e diferenças interindividuais), também interpreta a influência de dois tipos de preditores das mudanças observadas na performance dos adolescentes: a) um preditor dinâmico no tempo - a informação anualmente obtida da \%GTotal; e b) um preditor fixo no tempo - o gênero dos adolescentes (meninas codificadas com o número 0 , meninos com o 1 ).

Mediante a estrutura organizacional da informação foram colocadas quatro perguntas que reputamos de relevantes:

1. Será possível modelar a mudança no desempenho da força estática de preensão manual das quatro coortes de adolescentes seguidas de modo longitudinal-misto dos 10 aos 18 anos? A resposta positiva implica uma ligação das coortes numa trajetória típica, refletindo o comportamento médio do desenvolvimento da força estática dos músculos da preensão manual.

2. Qual o efeito do \%GTotal na performance do teste de preensão manual desses adolescentes, independentemente do seu gênero? Trata-se, aqui, 
de modelar o efeito de um preditor dinâmico no desenvolvimento da força.

3. Existem diferenças acentuadas entre gêneros no desempenho da força estática manual? Se sim, devem ser marcadas por trajetórias médias diferenciadas ao longo da idade.

4. Haverá uma forte variabilidade nas trajetórias de desenvolvimento da força estática manual nos adolescentes? Se ocorrer, deve ser referido pela expressão estatisticamente significativa da variância interindividual e intraindividual.

No sentido de responder às perguntas colocadas, o ajustamento dos modelos foi realizado no "software" HLM 7.01 ${ }^{20}$ através da técnica de estimação de máxima verossimilhança (do inglês "full information maximum likelihood"), suficientemente flexível para uso num delineamento longitudinal-misto. $\mathrm{O}$ processo de estimaçáo simultânea de todos os parâmetros dos vários modelos utiliza toda a informação disponível, assumindo que o padrão de casos omissos é "at random" (aleatório), uma consideração muito plausível, mas não tão exigente quanto a "missing completely at random"27-29. Para melhor descrever as trajetórias longitudinais individuais, trajetórias médias e respetivos preditores, foi adotado um procedimento "stepwise". Primeiramente, foi definido o submodelo do $1^{\circ}$ nível, utilizando-se uma função polinomial do tempo com complexidade crescente. Para ter uma primeira impressão do comportamento das medidas repetidas da preensão manual, os valores foram inicialmente representados num "spaghetti plot" providenciado pelo HLM (ver FIGURA 1, parte esquerda). Em seguida, baseado em sugestôes da literatura ${ }^{30-31}$ foram ajustados numa série de modelos polinomiais até o $3^{\circ}$ grau. A decisão final sobre o melhor modelo foi realizada de acordo com a "Deviance" (estatística que expressa a qualidade do modelo) e as correspondentes alteraçóes no valor de Qui-quadrado; o modelo mais complexo, i.e., com mais parâmetros, será melhor que o anterior se o valor da "Deviance" diminuir e esse decréscimo for estatisticamente significativo. Essa hipótese é testada pela estatística do Qui-quadrado e seus respetivos graus de liberdade, que são iguais à diferença entre o número de parâmetros estimados em cada modelo.
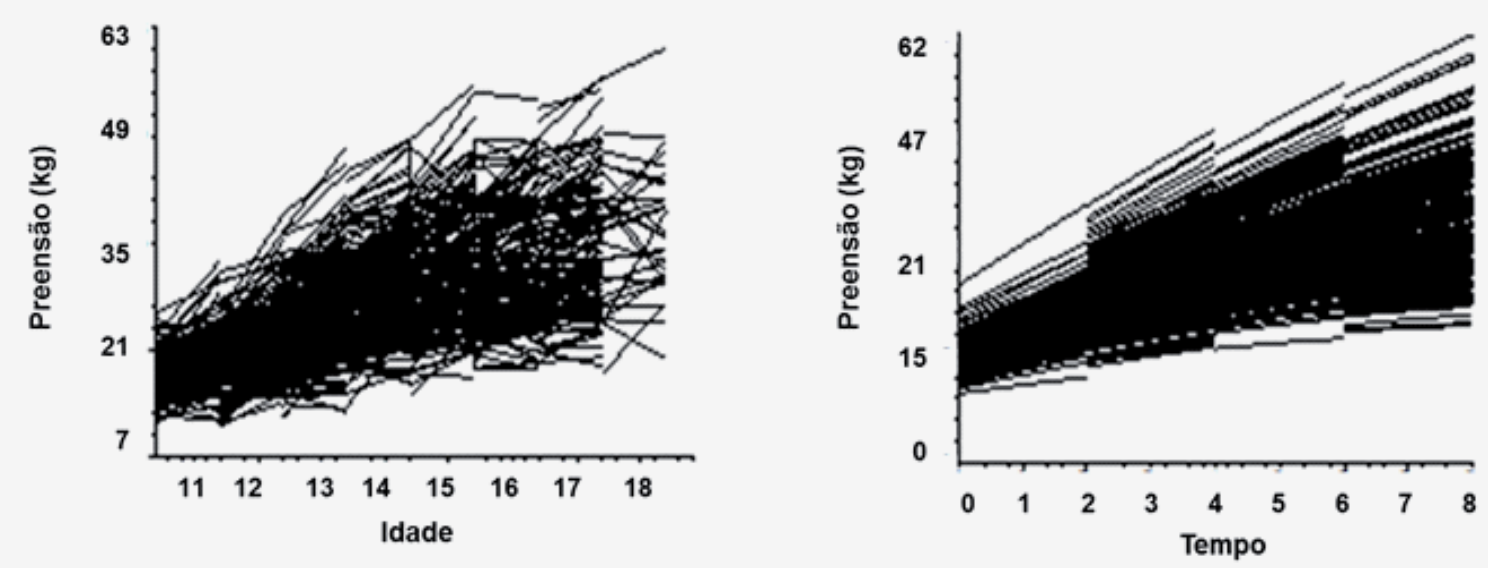

Parte esquerda: dados originais;

Parte direita: dados ajustados com uma nova métrica temporal.

FIGURA 1 - Representação do desempenho da força de preensão das quatro coortes de adolescentes em função do tempo ("outputs" do HLM 7.01).

O passo seguinte foi testar a importância da covariável dinâmica no tempo (\%GTotal), que é necessariamente incluída no submodelo do $1^{\circ}$ nível. Finalmente, foi incluído, no submodelo do $2^{\circ}$ nível o preditor fixo no tempo (gênero). Todos os parâmetros foram simultaneamente estimados e foi testada a sua significância em termos estatísticos. Para facilitar a interpretação dos resultados, sobretudo da dimensão temporal, é necessário efetuar uma alteraçáo da métrica temporal. Como cada coorte tem três avaliações no tempo (C1: avaliação 1,2 e 3; C2: avaliação 3, 4 e 5; C3: avaliação 5, 6 e 7; C4: avaliação 7,8 e 9), com um registro temporal em sobreposição, começamos a "contar o tempo do desenvolvimento da força estática” no início do estudo, i.e., na primeira avaliação da $\mathrm{C} 1=10$ anos. Deste modo o momento 0 corresponde aos 10 anos de idade. A métrica temporal do eixo X passou a ser: $0,1,2,3,4,5,6,7$ e 8 , que corresponde aos 10, 11, $12,13,14,15,16,17$ e 18 anos de idade. 


\section{Resultados}

Passamos, agora, a responder à primeira pergunta deste tutorial sobre a possibilidade de modelar a mudança no desempenho da força estática de preensão manual das quatro coortes de adolescentes. Na parte direita da FIGURA 1 e no Modelo 1 da TABELA 3 temos a resposta positiva. Com base na redução significativa $\left(\chi^{2}=53,412, p<0.001\right)$ da "Deviance" verificamos que um polinômio do $2^{\circ}$ grau se ajusta bem às trajetórias individuais. A interpretação dos resultados é a seguinte: a) o valor médio do desempenho da força de preensão manual de todos os adolescentes no "Baseline", i.e. aos 10 anos, é 15,660 \pm 0,148 kgf; b) o incremento médio anual de força estática manual é de 3,358 \pm 0,086 kgf/ano; c) há uma tendência para a estabilização dos ganhos, uma vez que a aceleração é negativa $\left(\beta=-0,089 \pm 0,012 \mathrm{kgf} / \mathrm{ano}^{2}\right)$.

A segunda pergunta de estudo procurava esclarecer acerca do efeito do \%GTotal na performance do teste de preensão manual dos adolescentes, independente do seu gênero. Os resultados deste segundo modelo estão na TABELA 3, mostrando um efeito negativo do \%GTotal no desempenho dos adolescentes $(\beta=-0,131 \pm 0,011 \mathrm{kgf} / \mathrm{ano})$. Isto significa que os adolescentes com percentual de gordura mais elevada têm, em média, menores incrementos anuais de força estática manual.
A terceira pergunta de estudo a ser respondida relacionava-se com a possível presença de diferenças entre gêneros no desempenho da força estática manual. A resposta está não só na FIGURA 2, que evidencia trajetórias médias distintas ao longo das idades, mas também nos resultados do Modelo 3 (ver TABELA 3), no qual se constata que: a) o valor médio de preensão manual das meninas no "Baseline" (i.e., aos 10 anos) é 16,392 \pm 0,188 kgf, sendo os meninos, em média, $2,370 \pm 0,252 \mathrm{kgf}$ mais fracos; b) a média de velocidade de ganhos anuais de força das meninas é de 2,563 $\pm 0,090$ $\mathrm{kgf} / \mathrm{ano}$; no entanto, os meninos têm ganhos superiores em 4,680 kgf/ano $(2,563 \pm 2,117) ; \mathrm{c})$ parece haver uma tendência para a estabilização dos incrementos de força estática manual $(\beta=-0,117 \pm$ $\left.0,011 \mathrm{kgf} / \mathrm{ano}^{2}\right)$. Chamamos a atenção do leitor que náo apresentamos um efeito aleatório na aceleraçáo (i.e., diferenças interindividuais na aceleração) por dois motivos: primeiro porque não há informação na literatura sobre esta matéria que justifique a sua inclusão (ver MaLINA et al..$^{30}$ ); segundo porque a sua inclusão não representava um ganho interpretativo - esta variância não era significativa, o algoritmo precisava de muitas interaçóes para convergir e a "Deviance" não era significativa.

TABELA 3 - Parâmetros ( \pm erro-padrão) dos modelos ajustados para o desempenho da força de preensão, com preditores variantes e invariantes no tempodo tempo ("outputs" do HLM 7.01).

\begin{tabular}{rccc}
\hline Parâmetros & $\begin{array}{c}\text { Modelo1 } \\
\text { (polinômio de 2ograu) }\end{array}$ & $\begin{array}{c}\text { Modelo 2 } \\
\text { (Modelo 1 + } \\
\text { efeito do \%GTotal) }\end{array}$ & $\begin{array}{c}\text { Modelo 3 } \\
\text { (Modelo 2 } \\
\text { + efeito do gênero) }\end{array}$ \\
\hline Efeitos fxos & & & \\
\hline "Baseline" & $15,660 \pm 0,148^{*}$ & $15,585 \pm 0,153^{*}$ & $16,392 \pm 0,188^{*}$ \\
Velocidade & $3,358 \pm 0,086^{*}$ & $3,429 \pm 0,086^{*}$ & $-2,370 \pm 0,252^{*}$ \\
Sexo & & & $2,563 \pm 0,090^{*}$ \\
Aceleraçáo & $-0,089 \pm 0,012^{*}$ & $-0,099 \pm 0,012^{*}$ & $2,117 \pm 0,061^{*}$ \\
Efeito do \%GTotal & & $-0,131 \pm 0,011^{*}$ & $-0,117 \pm 0,011^{*}$ \\
\hline Efeitos aleatórios & & & $0,025 \pm 0,012^{* *}$ \\
\hline Variância no "Baseline" & $9,511^{*}$ & $12,118^{*}$ & $14,205^{*}$ \\
Variância nas velocidades & $0,950^{*}$ & $0,820^{*}$ & $0,087^{*}$ \\
Variância residual & 8,858 & 8,852 & 8,883 \\
Número de parâmetros & 7 & 8 & 10 \\
"Deviance" & 58042,370 & 57478,232 & 55897,346
\end{tabular}



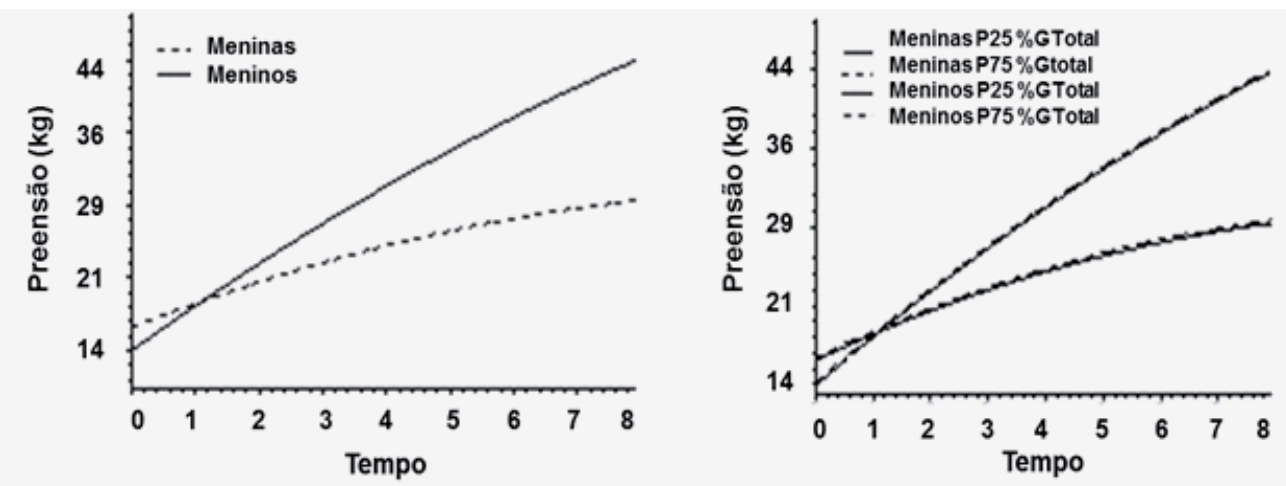

FIGURA 2 - Representação das trajetórias médias dos sujeitos dos dois sexos em função do tempo na parte esquerda da Figura. Na parte direita, temos as trajetórias em função, também, do Percentil 25 e 75 da \%GTotal ("output" do HLM 7.01).

Finalmente, a quarta pergunta tratava da identificação da forte variabilidade (i.e., variância estatisticamente significativa) nas trajetórias intraindividuais e nas diferenças entre sujeitos no desenvolvimento da sua força estática manual. Nos três modelos a componente aleatória mostra isso mesmo: variância significativa entre sujeitos (no "Baseline" e na velocidade), e intraindividual (variância residual) (TABELA 3).

\section{Discussão}

Este texto apresenta, na sua essência, um elucidativo ainda que breve sobre o uso esclarecido da MMN com informação proveniente de um delineamento longitudinal-misto. Para tanto servimo-nos da prova da força de preensáo manual cujos dados foram colhidos sucessivamente durante três anos em diferentes coortes de adolescentes dos 10 aos 18 anos. O recurso do delineamento em apreço permitiu a recolha mais célere da informação. Do mesmo modo, a metodologia impressa na MMN mostrouse suficientemente elegante e flexível para acomodar num modelo único, aspetos da mudança individual e das diferenças interindividuais do desempenho da força estática condicionado à ação do tempo.

As vantagens e utilidade do delineamento longitudinal-misto estão bem expressas na literatura internacional5,7-9,25,31-32. Um excelente exemplo do valor deste delineamento em produzir resultados estatísticos semelhantes ao de um delineamento longitudinal puro foi apresentado por DUNCAN et al. ${ }^{8}$ em 1996. Os autores compararam as trajetórias de mudança do uso de álcool de adolescentes dos 12 aos 17 anos, provenientes de dois processos de delineamentos - longitudinal puro e misto. No estudo longitudinal puro, uma coorte única de 81 adolescentes foi acompanhada durante seis avaliaçóes anuais consecutivas; no delineamento misto, quatro coortes de diferentes sujeitos $(n=461)$ foram avaliadas anualmente, durante três anos. Os modelos não diferiram significativamente nos resultados nem nas representaçóes gráficas. Adicionalmente, as análises mostraram efeitos similares das covariáveis utilizadas para predizer aspetos da mudança intraindividual e diferenças entre sujeitos. Num outro estudo longitudinal-misto de quatro anos, os mesmos autores ${ }^{32}$ examinaram a mudança nos níveis de atividade física de adolescentes, dos 12 aos 17 anos de idade, condicionados a fatores pessoais, familiares e dos pares de amigos. Os resultados mostraram um declínio significativo nos níveis de atividade física; adicionalmente ressaltaram a importância e o valor do delineamento utilizado para modelar a trajetória dos sujeitos em um menor espaço de tempo, minimizando algumas desvantagens do delineamento longitudinal puro.

Tal como referido anteriormente, para além da implementação de um "design" temporal que favoreça uma visão detalhada da mudança do fenômeno em estudo, é indispensável um modelo estatístico que possibilite a análise multivariada do processo de mudança ${ }^{14}$. O delineamento longitudinal-misto permite inúmeras possibilidades de análise de dados. Usualmente, há alguma tendência para recorrer a métodos analíticos tradicionais de efeitos fixos, como a regressão múltipla e análise de variância, que são limitados e restritivos no seu tratamento das medidas repetidas ${ }^{9,16,24}$, sobretudo por utilizarem somente informação completa, o que pode limitar 
o poder estatístico bem como a generalização das conclusóes. A MMN considera, simultaneamente efeitos fixos e aleatórios, é muito mais versátil, sendo ajustável à estrutura de dados provenientes de delineamentos longitudinais-mistos?: a) é suficientemente flexível para permitir a presença de informação omissa gerada pelo "design" misto ("missing by design"); b) os parâmetros estimados pelos modelos testados são mais precisos, visto que toda a informação disponível é considerada; c) permite o uso de preditores discretos e contínuos para modelar a mudança; d) o espaçamento temporal pode variar entre os sujeitos; e) permite a estimaçáo direta da correlação entre mudança e "status" inicial; ef) exibe um elevado potencial interpretativo da mudança intraindividual e das diferenças interindividuais associadas à influência de diferentes preditores 3,9,15,20,24.

No nosso exemplo, a MMN foi utilizada para ajustar modelos hierárquicos de natureza expansiva (i.e, com aumento no número de parâmetros) para modelar a mudança no desempenho de força de preensão manual. Na TABELA 3, de acordo com as diferenças significativas nas "Deviances" $\left(\chi^{2}=\right.$ $1580,885, \mathrm{p}<0,001$ ), fica evidente que cada modelo foi melhor que o anterior para descrever a complexidade dos dados. Os resultados do último modelo mostraram que no início do estudo as meninas tinham, em média, mais força estática que os meninos; no entanto, estes apresentaram maiores ganhos anuais de desempenho, superando as meninas ao longo do tempo. Adolescentes de ambos os sexos apresentaram trajetórias intraindividuais de mudança curvilíneas, havendo uma tendência de estabilização dos ganhos, uma vez que a aceleração foi negativa. A associação do \%GTotal com a força de preensão foi positiva ao longo de toda a trajetória de mudança da preensão.

Informaçóes longitudinais sobre o desempenho motor de adolescentes são em sua maioria datadas; contudo, apresentam descriçóes e interpretações importantes acerca da mudança condicionada pelo tempo (i.e., pela idade cronológica). Tal como salientado no nosso exemplo, MaLIna et al. ${ }^{30}$ relatam a existência de diferenças significativas entre sexos no desempenho da força estática entre os seis e os 18 anos. Os dados apresentados por estes autores retratam um aumento linear de força de acordo com a idade até os 13 ou 14 anos nos meninos, momento em que há uma aceleração no desenvolvimento da força; nas meninas o aumento é linear até cerca dos 16 ou 17 anos, sem evidências claras de um estirão. Embora pequena, a diferença entre sexos é significativa durante a infância, favorecendo sempre os rapazes. As diferenças mais expressivas ocorrem sobretudo após o período pubertário, com a marcada aceleraçáo no desenvolvimento de força dos meninos. Esta vantagem de desempenho dos meninos não foi observada no baseline do nosso estudo, podendo estar relacionada com o período pré-pubertário das meninas, que geralmente começa aos $10 \mathrm{anos}^{30}$. Nesse mesmo sentido, a análise cuidadosa da informação recentemente disponibilizada por SiLVA et al. ${ }^{31}$, de crianças e jovens da região norte do Brasil, mostra um quadro semelhante, meninas com maturação avançada apresentam valores de preensão manual superiores a meninos com maturação on time, entre aproximadamente os 10 e 13 anos de idade; as meninas com maturação on time têm valores semelhantes aos meninos entre os 10 e 12 anos.

O \%GTtotal foi um preditor dinâmico positivo da força de preensão. Apesar do valor da estimativa do parâmetro ser muito reduzido $(\beta=0,025 \pm 0,012$ $\mathrm{kgf} / \mathrm{ano}$ ), é significativo indicando que adolescentes com maior percentual de gordura têm, em termos absolutos, melhores desempenhos de força de preensão manual. Mais uma vez, o estudo de SiLva et al. ${ }^{31}$ vai ao encontro desses resultados, ao referir que a massa gorda corporal (mensurada pela soma de pregas subcutâneas) é um preditor positivo no desempenho da força estática manual. Este efeito positivo faz sentido, uma vez que estamos tratando de valores absolutos de força isométrica, que dependem do tamanho corporal dos sujeitos, como é bem conhecido na literatura ${ }^{30}$.

Para além da interpretação dos efeitos fixos, os efeitos aleatórios do nosso modelo merecem interpretação. O leitor verificou que a variância na velocidade de ganhos diminuiu do Modelo 1 para o Modelo 3 (ver TABELA 3), o que permite interpretar o "poder explicativo" do \%GTotal e do gênero nas diferenças de desempenho entre sujeitos. Uma forma de interpretar a relevância desses preditores é contrastar a magnitude das variâncias. Deste modo, do modelo não condicionado (i.e, sem preditores) para o modelo condicionado pelo \%Gtotal, temos o seguinte cálculo: $[(0,950-0,820) / 0,950]=0,136$. Isso significa que o preditor dinâmico explicou $13,6 \%$ da variabilidade nos ganhos de força estática dos adolescentes. Ao inserirmos o preditor fixo (sexo) no modelo final, temos: $[(0,820-0,087) / 0,820]=$ 0,894; uma explicação de $89,4 \%$ da variância na velocidade. A diferença entre $89,4 \%$ e $13,6 \%$ é de $75,8 \%$, e indica, essencialmente, a grande relevância desses dois preditores na identificação das diferenças de ganhos entre os sujeitos. 
De modo contrário, a variância no "baseline" não diminiu, apesar dos modelos condicionados serem significativos. Com a inclusão dos preditores (\%GTotal e gênero), os adolescentes se tornaram ainda mais diferentes em seus valores iniciais. Esse fato, seguramente, reflete a urgência em incluir outras variáveis correlatas da mudança do desempenho de força estática, como por exemplo, o "status" maturacional. Adicionalmente, a presença de variabilidade residual remanescente reforça a necessidade de maior esforço na modelação das trajetórias para explicar a heterogeneidade intraindividual da mudança.
Em conclusão, este estudo reforçou a validade e flexibilidade do uso do delineamento longitudinalmisto em pesquisas com informação repetida no tempo, sobretudo quando associado a técnicas robustas de análise, como a MMN. Esta metodologia analítica foi suficientemente flexível e altamente versátil na interpretação de aspectos da mudança individual e das diferenças interindividuais do desempenho da força estática ao longo do tempo. É claro que este é apenas um exemplo da enorme aplicabilidade deste modelo, que pode e deve ser estendido a diferentes domínios da Educação Física e Ciências do Esporte.

\begin{abstract}
Multilevel modeling and mixed longitudinal design in the research in Physical Education and Sport Medicine

The main aim of this study was to introduce the use of multilevel modeling in the data from a mixed longitudinal study concerning growth, development and health of Portuguese adolescents. The sample comprises 5155 subjects from 4 age cohorts: 10 to 12,12 to 14,14 to 16 and 16 to 18 years. Variables chosen for analysis were handgrip strength and percentage of total body fat. Multilevel modeling analysis showed a quadratic trajectory of adolescents' handgrip strength performance, with higher annual increasing in boys. The percentage of total body fat was positively associated with the static strength and there were marked interindividual differences. The use of a mixed longitudinal design allowed a faster data collection process; in addition, multilevel modeling analysis was sufficiently flexible and robust to accommodate, in a single model, aspects of individual and interindividual changes in static strength development over the time.
\end{abstract}

KEY WoRds: Longitudinal studies; Hierarchical modeling; Change; Motor performance.

\title{
Referências
}

1. Baltes P, Nesselroade J. History and rationale of longitudinal research. In: Nesselroade JB, editor. Longitudinal research in the study of behavior and development. Michigan: Academic Press; 1979.

2. da Silva S, Beunen G, Freitas D, Maia J. Estudos longitudinais sobre o crescimento somático e desempenho motor: delineamentos, desafios e necessidades. Rev Bras Cineantropom Desempenho Hum. 2013;15:130-43.

3. Maia J, Basso L, Oliveira J, et al. O desafio da informação longitudinal: um passeio "guiado" sobre modelação hierárquica, "tracking" e informação omissa com um conjunto de dados do estudo de Muzambinho. Rev Bras Edu Fís Esporte. 2010;24:413-31.

4. Taris TW. A primer in longitudinal data analysis. London: Sage; 2000.

5. Anderson ER. Analyzing change in short-term longitudinal research using cohort-sequential designs. J Consult Clin Psychol. 1993;61:929-40.

6. Bell RQ. Convergence: an accelerated longitudinal approach. Child Dev. 1953;24:145-52.

7. Bell RQ. An experimental test of the accelerated longitudinal approach. Child Dev. 1954;25:281-6.

8. Duncan S, Duncan T, Hops H. Analysis of longitudinal data within accelerated longitudinal designs. Psychol Methods. 1996;1:236-48. 
9. Duncan S, Duncan T. Accelerated longitudinal designs. In: Laursen B, Litlle T, Card N, editors. Handbook of developmental research methods. New York: Guilford Press; 2012. p.31-45.

10. Freitas D, Maia J, Beunen G, et al. Crescimento somático, maturação biológica, aptidão física, actividade física e estatuto socioeconómico de crianças e adolescentes madeirenses: o estudo de crescimento da Madeira: SAEFD. Funchal: Universidade da Madeira; 2002.

11. Maia J, Lopes V. Um olhar sobre criança e jovens da Região Autónoma dos Açores: implicações para educação física, desportos e saúde. Porto: FCDEF; 2003.

12. Basso L, Meira C, Oliveira J, et al. Crescimento e desenvolvimento motor de escolares de Muzambinho: um estuco com implicaçóes acadêmicas, sociais e de política interinstitucional. Rev Port Cien Desp. 2009;9:247-57.

13. Biassio L, Matsudo S, Matsudo V. Impacto da menarca nas variáveis antropométricas e neuromotoras da aptidão física, analisado longitudinalmente. Rev Bras Ciênc Mov. 2004;12:97-101.

14. Collins LM. Analysis of longitudinal data: the integration of theoretical model, temporal design, and statistical model. Annu Rev Psychol. 2006;57:505-28.

15. Bryk A, Raudenbush S. Application of hierarchical linear models to assessing change. Psychol Bull. 1987;101:147-58.

16. Francis DJ, Fletcher JM, Stuebing KK, Davidson KC, Thompson NM. Analysis of change: modeling individual growth. J Consult Clin Psychol. 1991;59:27-37.

17. Maia J, Garganta R, Seabra A, et al. Dados longitudinais e modelação hierárquica: um tutorial para investigadores das Ciências do Desporto. Rev Bras Cineantropom Desempenho Hum. 2005;7:94-108.

18. Fausto M, Carneiro M, Antunes C, Pinto J, Colosimo E. O modelo de regressão linear misto para dados longitudinais: uma aplicação na análise de dados antropométricos desbalanceados. Cad. Saúde Públ. 2008;24:513-24.

19. AAHPER. American Alliance for Health Physival Education and Reacreation. Youth fitness test manual. Washington: AAHPER; 1976.

20. Raudenbush S, Bryk A, Cheong Y, Congdon R, du Toit M. HLM 7: Hierarchical linear \& nonlinear modeling. Lincolnwood: Scientific Software International; 2011.

21. Raudenbush S, Bryk A. Hierarchical linear models: applications and data analysis methods. 2nd ed. Thousand Oaks: Sage; 2002.

22. Courgeau D. Methodology and epistemology of multilevel analysis: approaches from different social sciences. Dordrecht: Kluwer Academic; 2003.

23. Snijders T, Bosker R. Multilevel analysis: an introduction to basic and advanced multilevel modeling. Los Angeles: Sage; 2012.

24. Hernández-Lloreda M, Colmenares F, Arias R. Application of hierarchical linear modeling to the study of trajectories of behavioural development. Anim Behav. 2003;66:607-13.

25. Raudenbush S, Chan W. Growth curve analysis in accelerated longitudinal designs. J Res Crime Delinq. 1992;29:387-411.

26. Hofmann D, Jacobs R, Baratta J. Dynamic criteria and the measurement of change. J Appl Psychol. 1993;78:194-204.

27. Anderson ER. Accelerating and maximizing information from a short-term longitudinal research. In: Gottman J, editor. The analysis of change. Mahwah: Lawrence Erlbaum; 1995. p.139-64.

28. Hedeker D, Gibbons R. Longitudinal data analysis. New Jersey: John Wiley; 2006.

29. Raudenbush S. Hierarchical linear models to study the effects of social context on development. In: Gottman J, editor. The analysis of change. Mahwah: Lawrence Erlbaum; 1995. p.165-202.

30. Malina R, Bouchard C, Bar-Or C. Growth, maturation and physical activity. 2nd ed. Champaign: Human Kinetics; 2004.

31. Silva S, Beunen G, Claessens A, et al. Development of strength parameters during childhood and youth: a longitudinal study from Cariri, Brazil. In: Katzmarzyk P, Coelho e Silva M, editors. Growth and maturation in human biology and sports: festschrift honoring Robert M Malina by fellows and colleagues. Coimbra: Universidade de Coimbra; 2013. p.71-86.

32. Duncan SC, Duncan TE, Strycker LA, Chaumeton NR. A cohort-sequential latent growth model of physical activity from ages 12 to 17 years. Ann Behav Med. 2007;33:80-9. 


\section{Agradecimentos}

A todos os profissionais de Educação Física e Desporto envolvidos no estudo; à Fundação para Ciência e Tecnologia (FCT) de Portugal (financiamento ao projeto de referência PTDC/DES/67569/2006); à Coordenação de Aperfeiçoamento de Pessoal de Nível Superior - CAPES (bolsa de Doutorado pleno no exterior) e ao Conselho Nacional de Desenvolvimento Científico e Tecnológico - CNPq- (financiamento 305365/2011-8). Um agradecimento especial a todos os adolescentes participantes do estudo. 